\title{
Business Classification Based Adaptive Power Control Algorithm for Wireless Sensor Networks in Intelligent Electricity
}

\author{
Cui CAN, Wang $\mathrm{QI}^{*}$, Lu JUN
}

\begin{abstract}
WSN (Wireless Sensor Network) is an important way to transmit intelligent electricity business data, but it is more and more difficult to meet different performance requirements of different businesses. In the view of WSN power control problem, an adaptive power control algorithm based on intelligent electricity business classification was proposed in this paper. Firstly, the theory of node power control was analyzed. Secondly, the business classification and interference power adjustment factors were increased, to combine intelligent electricity business with network topology of WSN and realize the modeling of business classification based adaptive power control algorithm. Finally, this algorithm was compared with optimal power algorithm and LINT algorithm by simulation experiment in the performance of packet loss rate, delay and throughput. Simulation results showed that the algorithm proposed in this paper is better than others.
\end{abstract}

Keywords: adaptive power control; business classification; intelligent electricity; WSN

\section{INTRODUCTION}

WSN has been widely used in intelligent electricity data transmission, but technical requirements of QoS (Quality of Service) for different electrical businesses are still a problem to be solved [1-3]. For example, metering data for billing requires higher accuracy while demand response data needs to be transmitted faster; this is impossible for WSN with invariable transmit power. Power control of WSN nodes is an important factor in optimizing network performance, including extending network life cycle, routing topology optimization, improving communication capacity and so on [4]. Adjusting transmission power of WSN nodes is a feasible way to meet the performance requirements. As a result, researching on node power control has become a pop area of WSN $[5,6]$.

Existing WSN power control methods can be divided into three categories which contain data link layer, network layer and cross layer power control methods. The data link layer power control methods in $[7,9,10]$ mainly research on how to improve the spatial reuse of the WSN network with the given maximum transmission power of WSN nodes. For example, PCSMAC [9] is a typical power control algorithm with dormancy mechanism. Network energy efficiency has been improved greater by the algorithm than by traditional protocol. Network layer power control methods [11-14] adjust transmission power based on network node distribution, to connect the network with minimum transmitting power and improve network performance at the same time. For example, optimal connectivity power control [11] was proposed as a stable clustering algorithm (SSOC) based on optimal connectivity power. This algorithm changed node transmit power according to the number of neighbor nodes, and realized optimization of network throughput by using an effective variable power modulation technique. Cross layer power control algorithms $[8,10,16]$ formulate appropriate strategies by sharing network information between different layers. For example, CPC-AODV [10] (Ad hoc on-demand distance vector) combined power control method and routing algorithm, to reduce energy consumption and improve network throughput. Existing power control algorithms could improve network communication performance to a certain extent, but they are not suitable when transmitting different electricity business data. As mentioned above, WSN for intelligent electricity transmit different kinds of business data instead of cyclical power consumption data only. Load control data, for example, needs to be sent faster than normal data to balance power load immediately.

Aiming at above defects, an adaptive WSN power control algorithm for a different intelligent business is studied in this paper to meet QoS requirements of a different intelligent electricity business, which contains electric charge metering, load control, demand response, home energy management and so on, by power optimization of WSN nodes. Firstly, WSN power control theory is analyzed. On this basis, adaptive power control algorithm is proposed and realized. In addition, simulation experiment results compared with other traditional methods are finally given.

\section{ANALYSIS OF WSN POWER CONTROL THEORY}

WSN topological structure can be represented as a graph $G=(V, E)$, where $V$ is a collection of all nodes in WSN, and $E$ is the link collection between any two nodes in WSN. The following assumptions are made in this paper: (1) every node knows position of itself and other nodes in the network by using GPS or RSSI positioning technique; (2) all nodes in the network are stationary; (3) free space signal attenuation model $P \propto d^{n}$ was applied in this paper, where $d$ is Euclidean distance between send and receive nodes, $n$ is consumption index between two and four; (4) nodes can adjust their transmission power and range according to actual demand.

Before analyzing WSN power control principle, energy consumption model of WSN is introduced. We assume that $n$ nodes are placed in network area randomly, all nodes work in peer to peer mode in the process of routing establishment and data transmission. Communication radius can be adjusted by changing transmission power [9].

Energy consumption $E_{t x}$ to send a data packet of $k$ bit to a neighbor node $d$ meters away is shown as Eq. (1).

$E_{t x}(k, d)=\left\{\begin{array}{l}k \times E_{\text {elec }}+k \times \varepsilon_{f s} \times d^{2},\left(d<d_{0}\right) \\ k \times E_{\text {elec }}+k \times \varepsilon_{\text {amp }} \times d^{4},\left(d \geq d_{0}\right)\end{array}\right.$ 
Energy consumption of the neighbor node to receive the packet is shown as Eq. (2).

$$
E_{r x}(k)=k \times E_{\text {elec }}
$$

where, $E_{\text {elec }}$ means transmitting circuit loss, $\varepsilon_{f s}$ and $\varepsilon_{a m p}$ are power gains of two different patterns respectively, $d_{0}$ is the maximum transmission distance.

Furthermore, optimum transmit power for WSN node in free space can be calculated based on above theory. By referring to existing studies, received power $\mathrm{P}_{\mathrm{r}}$ is shown as follows.

$$
P_{r}=\frac{P_{t} G_{t} G_{r} \lambda^{2}}{4 \pi^{2} d^{2} L}
$$

$P_{t}$ is transmit power, $G_{t}$ and $G_{r}$ are transmit and receive antenna gain respectively, $\lambda$ is wavelength, parameter $d$ is Euclidean distance between transmitter and receiver, parameter $L$ is system loss factor which is greater than 1 .

Minimum threshold of receive power is set as $P_{t h}$, this means data packets received with lower power than $P_{t h}$ will be ignored. Thus, minimum transmit power, the minimum power to transmit data packets successfully, can be calculated and shown in Eq. (4).

$$
P_{\min }=\frac{P_{t h} 4 \pi^{2} d^{2} L}{G_{t} G_{r} \lambda^{2}}
$$

According to Eq. (3) and Eq. (4), minimum transmission power $P_{\min }$ can be further shown as Eq. (5).

$$
P_{\min }=\frac{P_{t} R_{t h}}{P_{r}}
$$

Considering wireless propagation loss, the minimum power is usually multiplied by a coefficient $c$ which is greater than one. So, we can obtain the optimal transmission power of WSN nodes in practice shown in Eq. (6)

$$
P_{o c}=c P_{\min }=c \frac{P_{t} R_{t h}}{P_{r}}
$$

To minimize energy consumption of WSN, it is necessary to change transmission power in data link layer by using smaller power. In data transmission process, nodes could learn transmitting power and receive power information from data packets, to make sure that optimal transmission power will be used when communicating with their neighbors.

\section{BUSINESS CLASSIFICATION BASED ADAPTIVE POWER CONTROL ALGORITHM FOR WSN}

\subsection{Basic Idea}

In this section, a business classification based adaptive power control algorithm (BC-APCA) for WSN is proposed by combining DiffServ and electric business characteristics. This algorithm belongs to cross layer power control method, including four parts: electric service classification, network topology information feedback, and node power adjustment strategy, as shown in Fig.1.

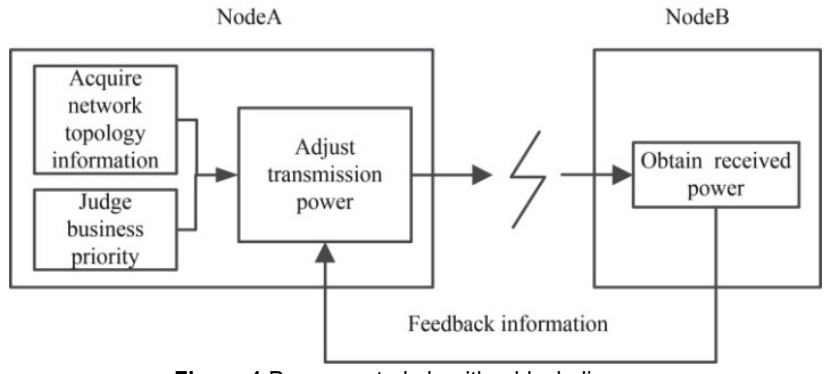

Figure 1 Power control algorithm block diagram

\subsection{Algorithm Modeling of Adaptive Power Control Based on Business Classification}

In BC-APCA, interference factor and business classification factor are introduced into WSN node optimal transmission power model, to change node power in different position when sending different business data. Related to traditional power control method, QoS requirement of different business and influence of node interference on network performance are considered in BC-APCA. The two kinds of factors will be given detailed introduction as below.

\subsubsection{Interference Factor for Power Control}

Node communication model in WSN is shown as Fig. 2. Distance between node $\mathrm{A}$ and sink node is $d_{i}, R_{i}$ is transmission radius of node $\mathrm{A}$, and its transmission area is $S_{i .} d$ is the distance from sink node to the most remote transmitting range of node $\mathrm{A}$, so $d=d_{i}+R_{i}, S$ is area of whole network.

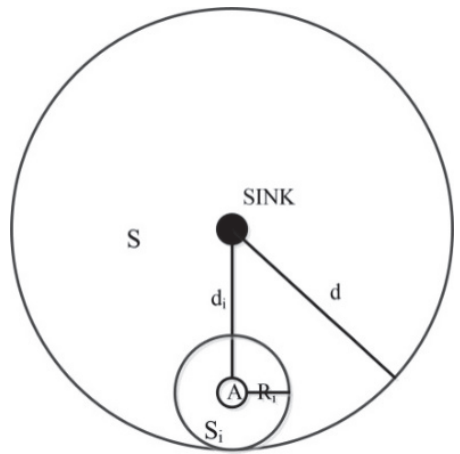

Figure 2 Model of jamming power adjustment factor node

In Fig. 2, $S_{i}$ is the sending area, which can be expressed by Eq. (7). Also whole area $S$ can be expressed by Eq. (8).

$S_{i}=\pi R_{i}^{2}$

$S=\pi d^{2}=\pi\left(d_{i}+R_{i}\right)^{2}$

In WSN, node communication efficiency is influenced by others. Large communication distance often causes high interference and low network performance. Therefore, node interference factor in the network where node 
transmission range is $R_{i}$ can be defined as $C_{d}$, which is shown as Eq. (9).

$$
C_{d}=\frac{S_{i}}{S}=\frac{\pi R_{i}^{2}}{\pi\left(d_{i}+R_{i}\right)^{2}}=\frac{R_{i}^{2}}{\left(d_{i}+R_{i}\right)^{2}}
$$

We can find that $C_{d}$ is proportional to $R_{i}$, and inversely proportional to $d_{i}$. In other words, when transmission radius increases, the interference factor becomes greater; but when the node is farther away from sink node, the interference factor becomes smaller.

\subsubsection{Business Classification Factor for Power Control}

In order to ensure QoS requirement of different electric business, business classification factor is necessary in BCAPCA. In WSN for intelligent electricity, nodes transmit electric power consumption data to sink periodically. Most of network resource is occupied to send huge amount of measurement data. But some other control information, such as load regulation message of demand response, also need to be sent by electric communication network in time. These two kinds of electric business are mainly considered in this study to meet different QoS requirements. Generally, burst data like load control data have higher priority in terms of delay than cyclical data. In this way, BC-APCA is also effective in other business scenarios. Business priority factor $C_{q}$ is defined as shown in Eq. (10).

$C_{q}=\left\{\begin{array}{l}0, \text { ordinary business } \\ 1, \text { high priority business }\end{array}\right.$
The value of $C_{q}$ of high priority business is 1 , and of ordinary business is 0 . This means high priority business data need higher network priority than normal data. Different kinds of data will be sent in different power according to priority factor in BC-APCA to get superior network performance.

\subsubsection{Adaptive Power Control Model Based on Business Classification}

According to the optimal transmission power equation calculated by Eq. (6), nodes could ensure network connectivity and minimize energy consumption at the same time. But existing power control methods change transmission power of nodes based on network state or distance to their neighbors. Different kinds of business data are sent at the same power when the destination node remains unchanged. This is unsatisfactory in intelligent electricity. Considering interference factor and different business requirements for QoS, an adaptive power control model is proposed in this paper. The adaptive power in BCAPCA can be calculated as Eq. (11) shows.

$P=\left(C_{q}+\frac{1}{C_{d}}\right) \frac{P_{t} R_{t h}}{P_{r}}$

In BC-APCA, nodes transmit higher priority business data packet with higher power while ordinary business with lower power. In addition, higher power will be used when interference factor is smaller to obtain better performance, and lower power to reduce data loss caused by interference when interference factor is greater.

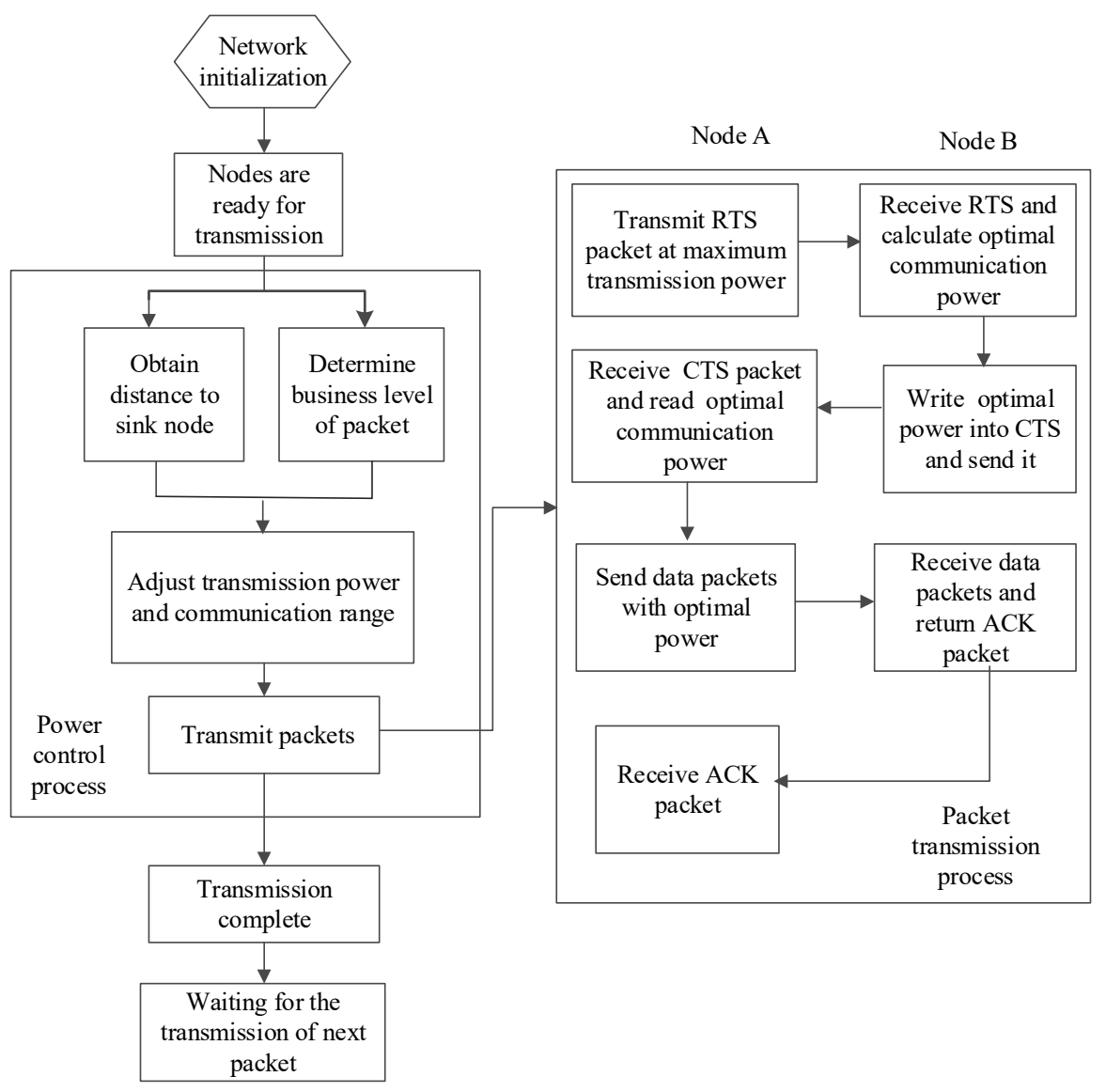

Figure 3 Flow chart of BC-APCA 


\subsection{Realization of Proposed Algorithm}

The detailed flow chart of BC-APCA mainly including power control process and data transmission process is shown as Fig. 3. In power control process, node obtaining information includes distance to sink node and priority of business data. Then send data at the transmit power calculated by Eq. (11). In data transmission process, node A (source node) firstly sends a RTS (Request to Send) packet to node $\mathrm{B}$ (next hop) at maximal transmission power so that node $B$ could calculate data transmission power by distance from node A, business level and network topology information in received RTS packet. Then node B sends a CTS (Clear to Send) packet back to node A. Node A gets transmission power information from CTS packet and begins to send data packets at this power. In the end, node B sends ACK (Acknowledgement) packet back to node A after receiving all data packets. Transmission process is completed only if the ACK packet is received by node A.

\section{SIMULATION EXPERIMENT RESULTS}

To verify performance of power control algorithm proposed in this paper, optimal power algorithm [5] and LINT [8] algorithm are compared with BC-APCA. The optimal power algorithm is a kind of data link layer power control method, where nodes transmit data with minimum power to neighbor nodes based on the position of nearest neighbor nodes, to minimize energy consumption. LINT algorithm is a network layer power control method where nodes adjust their transmission power to make sure the number of their neighbors is in a fixed range, so that network routing is optimized by power control.

OPNET14.5 is chosen as the simulation platform in this paper. Performance comparisons of three kinds of power control algorithms are analyzed in the same network structure. Simulation parameters are set as Tab. 1.

Table 1 Power control simulation parameters table

\begin{tabular}{|c|c|}
\hline Parameter type & Parameter value \\
\hline Network Area & $100 \times 100 \mathrm{~m}$ \\
\hline Node number & 100 \\
\hline Network topology & Tree network \\
\hline MAC protocol & IEEE 802.11 \\
\hline Packet size & 1024 bit \\
\hline Transmission interval & $0.1 \mathrm{~s}$ \\
\hline Simulation time & $200 \mathrm{~s}$ \\
\hline
\end{tabular}

Fig. 4 is a performance comparison chart of packet loss rate, where horizontal axis represents time (second), and vertical axis is packet loss rate (percent). As Fig. 4 shows, packet loss rate of BC-APCA is $16 \%$, which is significantly better than $22 \%$ of optimal power algorithm and $25 \%$ of LINT algorithm. This is because that network performance is seriously affected by interference and collision. BCAPCA takes interference factor into account and as a result, interference is eased and packet loss is reduced when nodes are transmitting data in WSN.

Fig. 5 is performance comparison of time delay, where horizontal axis represents time (second), and vertical axis represents network average time delay (millisecond). We can see that average delay of BC-APCA is $70 \mathrm{~ms}$, which is $30 \%$ and $55 \%$ less than optimal power algorithm and LINT algorithm respectively. This is also because the BC-APCA algorithm considers interference factor to reduce the number of retransmissions caused by interference and collision, so that time delay is significantly reduced. As time delay is an important index in intelligent electricity, and BC-APCA could meet real-time requirement of some electric business data especially event signals, it is more suitable to use BC-APCA in electric communication WSNs.

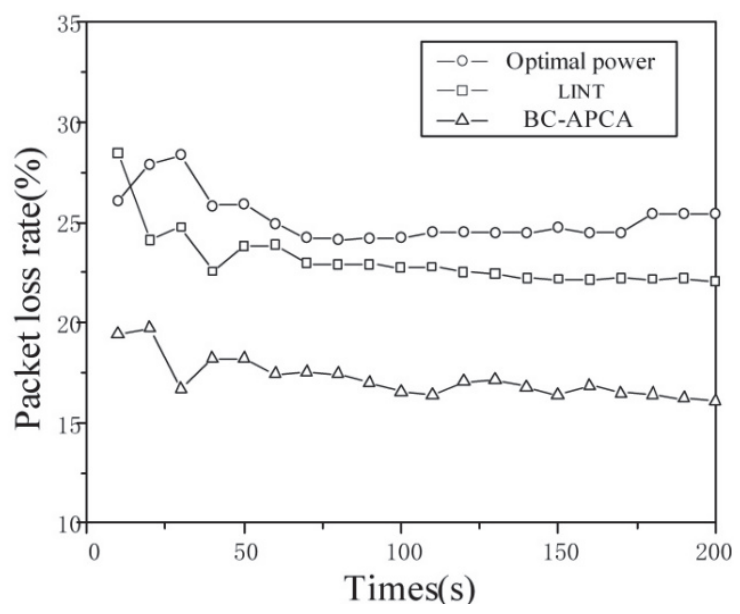

Figure 4 Comparison diagram of packet loss rate

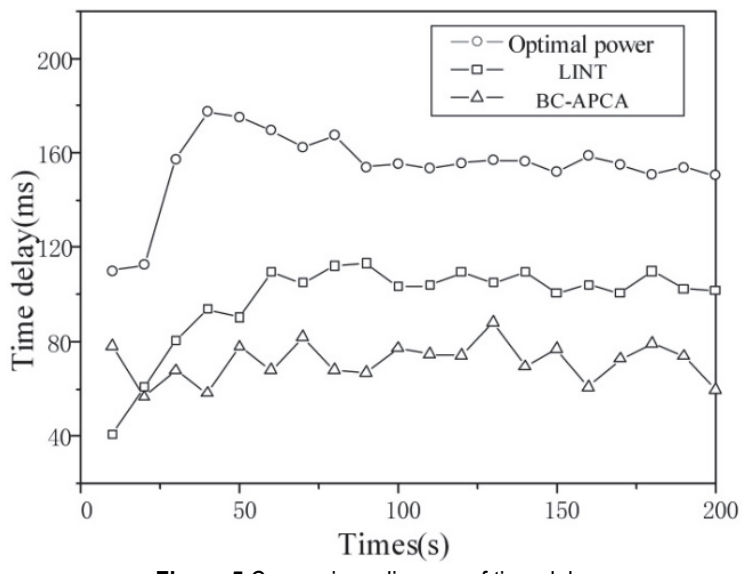

Figure 5 Comparison diagram of time delay

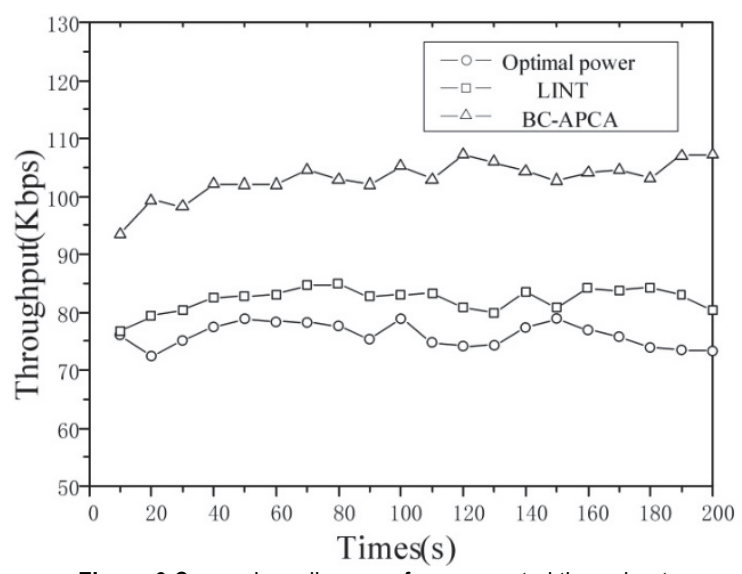

Figure 6 Comparison diagram of power control throughput

Fig. 6 is the throughput comparison of three algorithms, where horizontal axis is time (second), and vertical axis is throughput (kilobit per second). The average throughput of BC-APCA is $105 \mathrm{kBps}$, which is 1.3 and 1.5 times greater than optimal power algorithm and 
LINT algorithm. As interference factor and business classification factor are added into the power control model of BC-APCA algorithm to reduce the number of collisions and retransmissions, the network throughput is improved significantly. The priority factor is considered to meet the delay and throughput QoS requirements of different electricity businesses so that communication defects caused by other power control methods, like multi-hop delay of low power and interference collision of high power, are avoided in BC-APCA. It is necessary to send different business data at different transmission power in intelligent electricity to get better performance.

\section{CONCLUSIONS}

In order to meet QoS requirements of a different business in intelligent electricity on quality of services, a business classification based adaptive power control algorithm (BC-APCA) for WSN was proposed based on basic power optimization control model. The algorithm adjusted transmission power by improved power control model with interference and business priority factors to realized performance optimization of a different electricity business. Simulation results show that BC-APCA own better network performance than traditional power control algorithms in packet loss rate, time delay and throughput. As intelligent electricity business is changing and increasing rapidly, we will focus on the studies of WSN power control method for more kinds of electric business in the future.

\section{REFERENCE}

[1] Wang, H., Guan, Z., Yang, T. et al. (2014). Top-k query framework in wireless sensor networks for smart grid. China Communications, 11(6), 89-98. https://doi.org/10.1109/CC.2014.6879007

[2] Kreibich, O., Neuzil, J., \& Smid, R. (2014). Quality-Based Multiple-Sensor Fusion in an Industrial Wireless Sensor Network for MCM. IEEE Transactions on Industrial Electronics, 61(9), 4903-4911. https://doi.org/10.1109/TIE.2013.2293710

[3] Fateh, B., Govindarasu, M., \& Ajjarapu, V. (2013). Wireless Network Design for Transmission Line Monitoring in Smart Grid. IEEE Transactions on Smart Grid, 4(2), 1076-1086. https://doi.org/10.1109/TSG.2013.2241796

[4] Intanagonwiwat, C. et al. (2000). Directed Diffusion: A scalable and robust communication paradigm for sensor networking. Sixth Annual International Conference on Mobile Computing and Networking, 56-67. https://doi.org/10.1145/345910.345920

[5] Li, F. M., Xu, W. J., \& Liu, X. H. (2008). Power control for wireless sensor networks. Journal of Software, 19(3), 716732. https://doi.org/10.3724/SP.J.1001.2008.00716

[6] Akyildiz, I. \& Kasimoglu, I. (2004). Wireless sensor and actor networks: Research challenges. Ad Hoc Networks Journal (Elsevier), 2(4), 351-367. https://doi.org/10.1016/j.adhoc. 2004.04.003

[7] Jaffres Runser, K., Comaniciu, C., \& Gorce, J. M. (2009). UGRAB: A Utility-Based Gradient Broadcasting Algorithm for Wireless Sensor Networks. IEEE Military Communications Conference, 117-123. https://doi.org/10.1109/MILCOM.2009.5380037

[8] Jiang, J., Ji, P., Sun, Q. et al. (2013). A Power Control Based Cross-layer Communication Protocol for Wireless Sensor networks. Proceedings of the International Conference on
Computer, Networks and Communication Engineering (ICCNCE 2013), 30, 617-620. https://doi.org/10.2991/iccnce.2013.153

[9] Nar, P. C. \& Cayirci, E. (2005). PCSMAC: a power controlled sensor-MAC protocol for wireless sensor networks. EWSN'05, Piscataway: IEEE Computer Society, 81-92. https://doi.org/10.1109/EWSN.2005.1462001

[10] Huang, H., Hu, G., \& Yu, F. (2010). A routing algorithm based on Cross-layer Power Control in wireless ad hoc networks. Communications and Networking in China (CHINACOM), 2010 5th International ICST Conference on, 1-6. https://doi.org/10.4108/chinacom.2010.30

[11] Li, F.-M., Liu, X.-H., \& Kuang, H.-L. et al. (2009). Research on a stable clustering algorithm based on the optimal connectivity power for wireless sensor networks. Journal on Communications, 30(3), 75-83.

[12] Chen, Y., Chen, L., Rong, L. I. et al. (2009). An adaptive power control algorithm for wireless sensor networks using neural node. Engineering Journal of Wuhan University, 42(2), 256-259.

[13] Lui, Z. H., Correia, A., \& Daniel, F. (2007). Macedo. Transmission power control techniques for wireless sensor networks. Computer Networks, 51(6), 4765-4779. https://doi.org/10.1016/j.comnet.2007.07.008

[14] Li, P., Shen, Q., Fang, Y. et al. (2009). Power controlled network protocols for multi-rate ad hoc networks. IEEE Transactions on Wireless Communications, 8(4), 21422149. https://doi.org/10.1109/TWC.2009.080580

[15] Liu, X., Luo, J., \& Vasilakos, A. (2011). Compressed data aggregation for energy efficient wireless Sensor networks. 8th Annual IEEE Communications Society Conference on Sensor, Mesh and Ad Hoc Communications and Networks, 46-54.

[16] Liu, T., Li, Q., \& Liang, P. (2012). An energy-balancing clustering approach for gradient-based routing in wireless sensor networks. Computer Communications, 35(17), 21502161. https://doi.org/10.1016/j.comcom.2012.06.013

\section{Contact information:}

Cui CAN, PhD

China Electric Power Research Institute,

Haidian District, Beiijing, China, 100192

E-mail: cc52112345@163.com

\section{Wang Ql, PhD}

(Corresponding author)

China Academy of Information and Communications Technology,

Haidian District, Beijing, China

E-mail: qiuqian12390@126.com

Lu JUN, PhD, Associate Professor

North China Electric Power University,

Changping District, Beijing, China

E-mail: lujun@ncepu.edu.cn 